\title{
Self-assembled sub-wavelength structures and form birefringence created by femtosecond laser writing in glass: properties and applications
}

\author{
Peter G. KAZANSKY and Yasuhiko SHIMOTSUMA* \\ Optoelectronics Research Centre, University of Southampton, S017 1BJ, United Kingdom \\ *Innovative Collaboration Center, Kyoto University, Kyotodaigaku-Katsura Nishikyo-ku, Kyoto, 615-8520
}

Properties and applications of self-assembled sub-wavelength structures and related form birefringence produced by femtosecond laser writing in silica glass are reviewed.

(2008 The Ceramic Society of Japan. All rights reserved.

Key-words : Femtosecond laser writing, Laser processing, Nanostructuting, Self-assembly, Photosensitivity, Birefringence, Silica glass

[Received August 4, 2008]

\section{Introduction}

When an intense femtosecond laser pulse is focused into transparent material, e.g. silica glass, high-order nonlinear absorption allows the energy to be deposited predominantly within the focal volume, producing a local permanent refractive-index modification. ${ }^{1)}$ Although the process of energy absorption is now well understood, little is known about the actual formation of the directly written structures. ${ }^{2)}$ Depending on the level of laser intensity, one can induce any of three qualitatively different types of modification or damage in silica glass: low intensity induces a smooth positive refractive-index change relative to the unprocessed material (type I-fs); intermediate intensity results in birefringent regions (type II-fs); and at high intensity the damage consists of voids embedded into the glass (type III-fs). Type I modification has been used to achieve waveguides and couplers, ${ }^{1,3)}$ whereas type III-fs void like defects have been exploited for data storage and photonic crystals. ${ }^{4), 5)}$ The intermediate type II-fs regime has received little attention until now but indeed seems to have intriguing properties. Type II-fs structures were first observed in Ge-doped silica where they show an anisotropic light scattering that is dependent on the plane of polarization of the light. ${ }^{6}$ Anisotropic scattering has been interpreted in terms of photoelectrons moving along the direction of the light's polarization, inducing index inhomogeneities. Later uniaxial birefringence imprinted in structures written within fused-silica plates was observed. ${ }^{7), 8)}$ Anisotropic reflection was then observed and explained in terms of the formation of a self-organized nanograting within the focal volume, ${ }^{9)}$ and finally the existence of the self-induced nanograting was proved by direct imaging. ${ }^{10), 11)}$ During the energy absorption process, tunneling, multiphoton and avalanche ionization produce free electrons within the focus of the ultrashort pulsed laser. ${ }^{12)}$ The observations suggest that, in a certain intensity range, the interference between longitudinal electron plasma waves leads to the formation of nano-sized gratings with a pitch as small as $150 \mathrm{~nm} .{ }^{10)}$ These periodic structures are ruled in the direction parallel to the polarization of the writing laser and consist of thin regions of index of refraction $n_{1}$, characterized by a strong oxygen deficiency, ${ }^{10)}$ surrounded by larger regions of index $n_{2}$ (Fig. 1). Such a periodic assembly

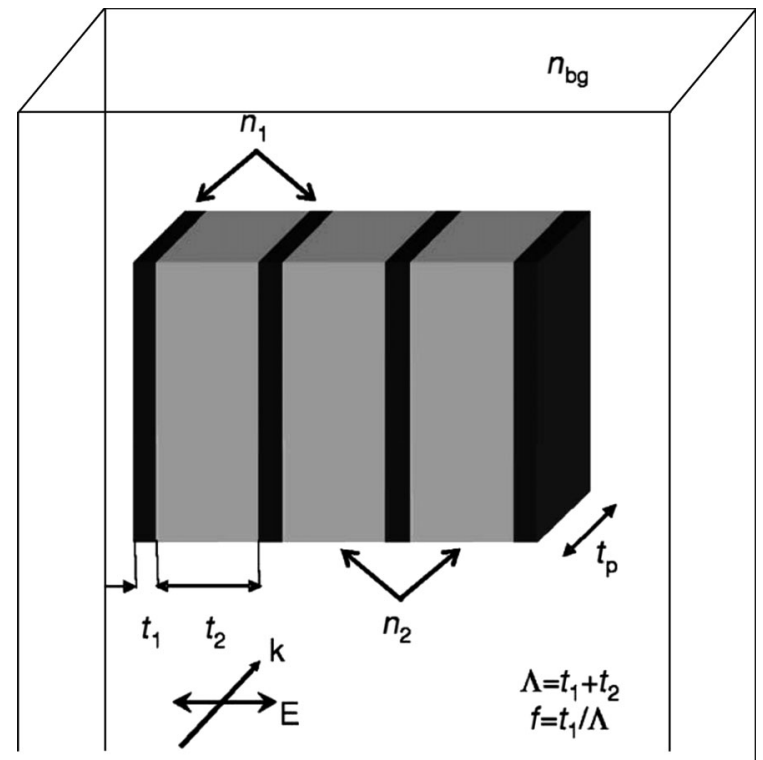

Fig. 1. Schematic of the self-assembled nano-grating formed in the irradiated volume of the directly written structures of type II-fs. $n_{\mathrm{bg}}$ : refractive index of silica, $n_{1}, n_{2}$ : local refractive indices of the nanograting, $f=t_{1} / \Lambda$ : filling factor, $\Lambda=t_{1}+t_{2}$ : period of the nanograting, $t_{1}, t_{2}$ : width of the regions with index $n_{1}$ and $n_{2}$, respectively.

behaves as a uniaxial negative birefringent material which is consistent with a planar form birefringence, whose optical axis is parallel to the direction of the polarization of the writing laser. It has been shown that the local refractive index change can be as high as -0.1 with respect to the unprocessed material, making these self-assembled structures the strongest laser written nanogratings ever observed. ${ }^{13)}$ Changes in the nanogratings due to variation of the pulse duration have been investigated. ${ }^{14)}$ The extraordinary stability of these nanogratings has also been reported. ${ }^{15)}$ Embedded micro-reflectors, ${ }^{9)}$ retardation plates, ${ }^{13)}$ and micro-fluidic channels ${ }^{16)}$ based on these nanostructures have been reported; however their optimization requires a systematic characterization of the properties of the periodic structures ver- 
sus various fabrication parameters. ${ }^{17)}$ More recently it has been reported a remarkable phenomenon manifested as a change in material modification, in particular the formation of nanostructures and related form birefringence by reversing the writing direction. ${ }^{18)}$

Here we review the results of the study of the self-assembled sub-wavelength structures and related form birefringence produced by femtosecond laser writing in silica glass.

\section{Characterization of self-organised structures and form birefringence}

An amplified, mode-locked Ti:sapphire laser operating at a wavelength tunable from 800 to $850 \mathrm{~nm}$, with a 200 -fs pulse duration and a $250-\mathrm{kHz}$ repetition rate, was utilized to effect all the direct-write processes. ${ }^{13)}$ The laser light was focused via either a $10 \times(\mathrm{NA}=0.21)$ or a $50 \times(\mathrm{NA}=0.55)$ objective to a focal spot of 4 or $1.5 \mathrm{~mm}$, respectively, into the sample, which was mounted upon a computer-controlled linear motor translation stage. Using this setup with the laser tuned at $835 \mathrm{~nm}$ and focused by the $10 \times$ objective, we directly wrote a matrix of $4 \times$ 4 quasi-uniform regions of $100 \mu \mathrm{m} \times 100 \mu \mathrm{m}$ each (sample 1 ) within the bulk of silica glass (Herasil 1). We constructed each processed zone by writing 100 adjacent lines spaced $1 \mu \mathrm{m}$ apart. To be able to find the transition between types I-fs and II-fs of damage, we wrote the micromachined zones with different laser dosages. Each row of the matrix of quasi-uniform regions was processed with constant average power $(P 1=160 \mathrm{~mW}, P 2=100$ $\mathrm{mW}, P 3=80 \mathrm{~mW}, P 4=60 \mathrm{~mW}$ ); each column, with the same number of pulses $\left(N 1=5 \times 10^{4}, N 2=2.5 \times 10^{4}, N 3=1.7 \times 10^{4}\right.$, $\left.N 4=1 \times 10^{4}\right)$. Figure 2 shows an image of the first two rows of the sample acquired between cross polarizers. Only the first row (P1) of the matrix, written at the highest intensity, shows the birefringence that we describe as type II-fs; the rest are of type I-fs. To facilitate the measurement of the refractive- index change of each zone with respect to the unprocessed bulk, we used an interferometric phasestepping technique. ${ }^{13)}$ Figure 3 shows a schematic of the interferometer with which we measured the laser-induced phase shift. The sample was positioned in the fixed arm of the interferometer, and we varied the reference arm by moving mirror M2 by using a computer-controlled piezoelectric actuator. A polarized $\mathrm{He}-\mathrm{Ne}$ laser beam was split by optical flat OF1at the input of the interferometer, recombined at OF2, and imaged by a charged-coupled device (CCD), which transferred the data to a computer. Intensity $I$ at the CCD camera was therefore given as

$$
I\left(x^{\prime}, y^{\prime}\right)-I_{0}(x, y)\left\{1+V \cos \left[\phi(x, y)+\phi_{0}(t)\right]\right\},
$$

where $(x, y)$ was the plane where the sample sat and $\left(x^{\prime}, y^{\prime}\right)$ was its conjugate plane at the CCD camera, $I_{0}$ was the bias in the interference fringe, and $V$ was the visibility of the fringes. $\phi_{0}$ was

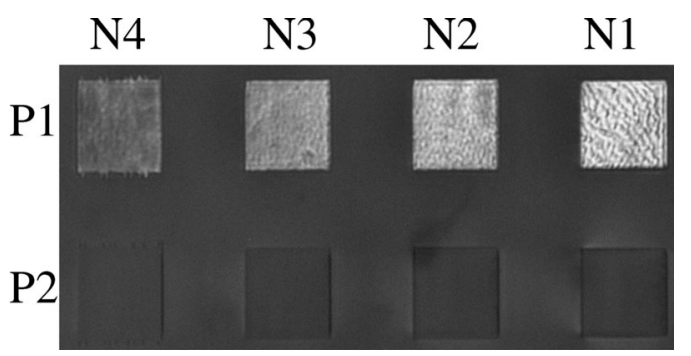

Fig. 2. Picture of part of sample 1 taken between cross polarizers. the variable phase, and $\phi$ was the additional unknown phase added by the sample. $\phi_{0}$ was stepped through approximately $2 \pi$ with a CCD image captured at each step. The intensity at each pixel of the CCD evolved according to Eq. (1), which we then fitted to extract relative phase $f$, resulting in the phase image shown in Fig. 4. This setup permitted a resolution of $\sim 5 \mu \mathrm{m}$ and a level of noise of $\pm 0.1 \mathrm{rad}$.

Figure 4 shows the phase images of the directly written sample for light travelling in the interferometer polarized parallel to the writing light $[x x$; Fig. 4(a)] and orthogonal to it [xy; Fig. 4(b)]. Table 1 lists the measured relative phase shift versus polarization for each region of the matrix, with strong birefringence apparent in $P 1$. To obtain more data we also analyzed the birefringent directly written Fresnel zone plates $\mathrm{A}$ and $\mathrm{B}$ previously reported ${ }^{8)}$ In this case we measured $\Delta \phi_{x x}=(-1.5,-1.3) \mathrm{rad}$, respectively, and $\Delta \phi_{x y} \sim-0.2 \mathrm{rad}$ for both plates. The striking result is that the $x x$-polarized light travelling into the samples sees a negative phase shift with respect to the light travelling into untouched silica. The $x y$-polarized light sees a small negative

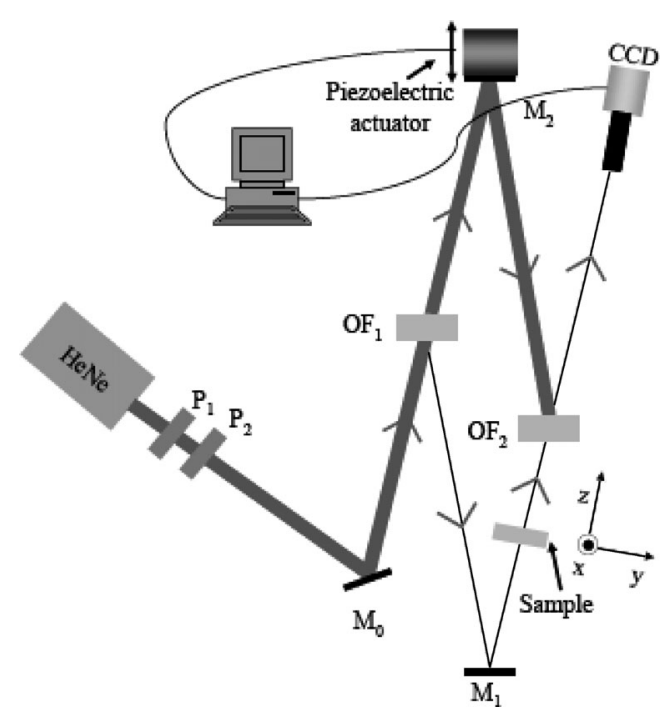

Fig. 3. Schematic of the interferometer used to measure the phase shift of the directly written sample with respect to the untouched bulk: M0M2, mirrors; P1, polarizer; other abbreviations defined in text.

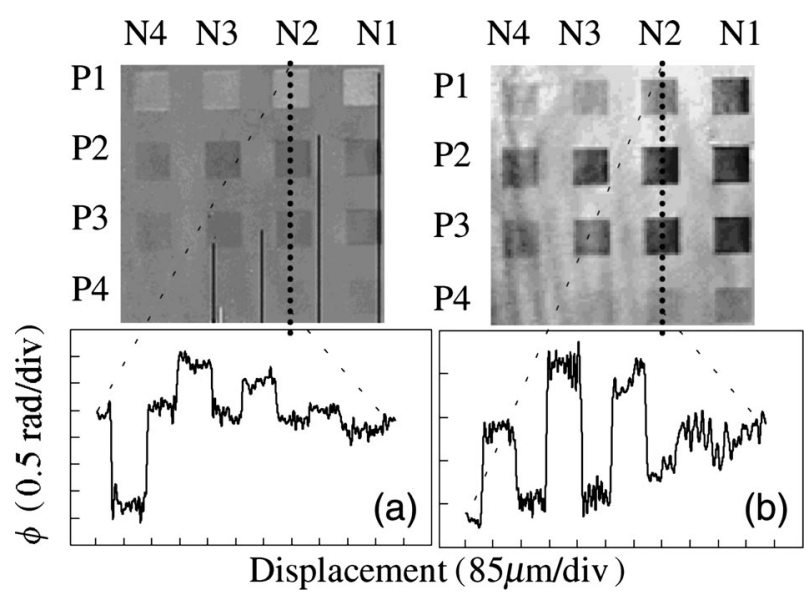

Fig. 4. Phase images of the directly written sample for two polarizations, (a) $x x$ and (b) $y y$, of the light travelling into the interferometer. 
Table 1. Phase Changes in the Micromachined Zones

\begin{tabular}{|c|c|c|c|c|c|c|c|c|}
\hline \multirow{2}{*}{$\begin{array}{l}\text { Average } \\
\text { Power }\end{array}$} & \multicolumn{4}{|c|}{$\Delta \phi_{x x}(\mathrm{rad})$} & \multicolumn{4}{|c|}{$\Delta \phi_{x y}(\mathrm{rad})$} \\
\hline & $N 4$ & $N 3$ & $N 2$ & $N 1$ & $N 4$ & $N 3$ & $N 2$ & $N 1$ \\
\hline$P 1$ & -0.8 & -0.8 & -1.6 & -2.0 & 0.1 & 0.5 & 0.8 & 1.3 \\
\hline$P 2$ & 0.8 & 1.2 & 1.2 & 1.2 & 0.7 & 1.2 & 1.4 & 1.5 \\
\hline$P 3$ & 0.6 & 0.8 & 1.0 & 0.8 & 0.5 & 1.0 & 1.2 & 1.2 \\
\hline$P 4$ & 0.1 & 0.1 & 0.2 & 0.4 & 0.1 & 0.2 & 0.4 & 0.5 \\
\hline
\end{tabular}

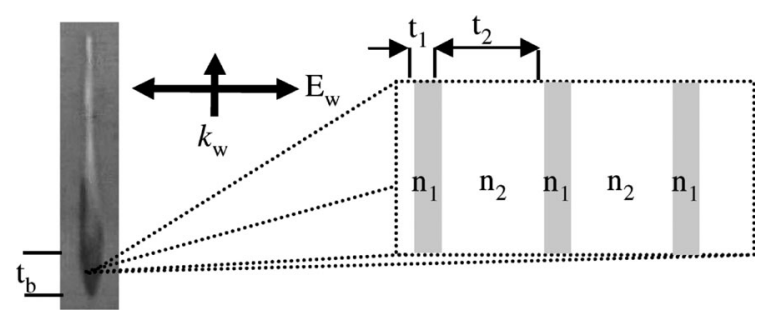

Fig. 5. Schematic of the self-organized nanograting induced in the glass. $E_{\mathrm{w}}$ and $k_{\mathrm{w}}$, electric field and wave vector, respectively, of the laser used to micromachine the glass.

index change in the Fresnel zone plates and a positive index change in sample 1. By applying a theoretical model of form birefringence to the directly written structures we can justify the negative index change as well as the possibility of a polarizationdependent sign of the effective index change. The inset of Fig. 5 shows an idealized schematic of the self-organized nanograting induced in the glass, where $\Lambda=t_{1}+t_{2}$ is the period of the nanostructure and $n_{1}$ and $n_{2}$ are the unknown local refractive indices. A backscattering scanning electron microscope image revealed that $\Lambda=300 \mathrm{~nm}$ and $f=\Lambda / t_{1}<0.2$ for Fresnel zone A. Such a grating, whose periodicity is smaller than the wavelength of the incident radiation, will produce no diffracted orders and will act as a birefringent material. When a monochromatic wave is incident upon the grating, the average index of refraction that the light will see will depend on the polarization direction of the light. ${ }^{19)}$ Figure 5 shows the typical shape of a femtosecond directly written structure in the same plane as of the propagation of the light. In agreement with what was reported by other authors, ${ }^{7}$ it was found that only a small percentage $t_{\mathrm{b}}$ of the overall thickness contributes to the birefringence; hence the model assumes that the nanograting is localized in $t_{\mathrm{b}}$. The phase shift measured with the interferometer is therefore given by

$$
\begin{aligned}
& \Delta \phi_{x y}-\frac{2 \pi}{\lambda} t_{\mathrm{b}}\left\{\left[\frac{n_{1}{ }^{2} n_{2}{ }^{2}}{f n_{2}{ }^{2}+(1-f) n_{1}{ }^{2}}\right]^{1 / 2}-n_{0}\right\}+\overline{\Delta \phi}, \\
& \Delta \phi_{x y}-\frac{2 \pi}{\lambda} t_{\mathrm{b}}\left\{\left[f n_{1}^{2}+(1-f) n_{2}{ }^{2}\right]^{1 / 2}-n_{0}\right\}+\overline{\Delta \phi},
\end{aligned}
$$

where $\lambda$ is the wavelength used in the interferometer, $n_{0}$ is the index of refraction of the unprocessed material, and $\Delta \phi$ is the phase shift given by the nonbirefringent tail of the written feature. Figure 6 shows in a contour plot the values of $\Delta \phi_{x x}$ (dashed-dotted curve), $\Delta \phi_{x y}$ (dotted curve), and $b=\Delta \phi_{x y}-\Delta \phi_{x x}$ (solid curve) calculated for $f=0.1$ and assuming that $t_{\mathrm{b}}=10 \mu \mathrm{m}$ and $\Delta \phi=0.1 \mathrm{rad}$. These functions are plotted versus the local refractive- index changes. The shaded area contains all the solutions for which $\Delta \phi_{x x}<0$ and $\Delta \phi_{x y}>0$. The curves that correspond

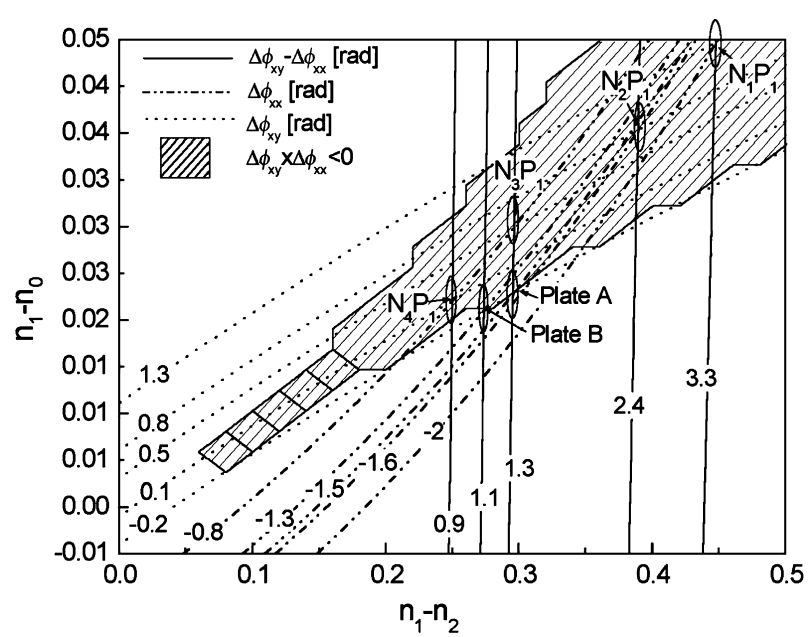

Fig. 6. Theoretical results when the model of form birefringence was applied to the directly written structures.

to various values of birefringence $b$ are almost vertical, suggesting that the birefringence depends mainly on the difference between $n_{1}$ and $n_{2}$. All the experimental solutions are highlighted by ellipses in the figure sized to include a variation in $\Delta \phi$ of as much as 1 order of magnitude larger or smaller. Assuming reasonable numbers, the experimental results match the theory for $n_{2}-n_{0}=(2$ to 5$) \times 10^{-2}$ and $n_{1}-n_{0}=(-4$ to -2$) \times 10^{-1}$, and it can therefore be concluded that locally the laser induces a periodic series of nanozones in which the material is denser than the substrate that alternates with other zones in which the material is more rarefied. More investigations are required, but a consistent model has been offered to explain birefringence, anisotropic reflection, and the negative index change shown by a directly written structure above a certain intensity threshold (type II-fs). Furthermore, the results shown in Table 1 demonstrate that a difference in phase of $\sim \pi$ between the effective phase shifts seen by two orthogonal polarizations was achieved. These properties may be used to fabricate embedded half-wave plates and polarization dependent devices such as retarders.

\section{Extraordinary stability of form birefringence pro- duced by femtosecond laser irradiation}

It has already been reported that the femtosecond direct written structures of type I-fs have a stability at elevated temperatures comparable to the strongest UV laser induced refractive index changes. ${ }^{20), 21)}$ It was also observed that the femtosecond laser written structures of type I-fs and type II-fs display the different response to increased temperature and in particular the structures of type II-fs demonstrate the extraordinary behavior, whose refractive index change increases with temperature. ${ }^{15)}$

An amplified, mode-locked Ti: Sapphire laser operating at a wavelength of $800 \mathrm{~nm}$, with $200 \mathrm{fs}$ pulse duration and $100 \mathrm{kHz}$ repetition rate, was utilized to fabricate the structures to be tested. The laser beam was linearly polarized and focused with a $10 \times$ objective. The femtosecond written sample consisted of an array of six square regions of side length of $100 \mu \mathrm{m}$, embedded in the bulk of fused silica (Herasil 1). The sample was mounted upon a computer controlled linear motor translation stage, which could move in the three directions with a spatial resolution of few nanometers. To be able to find the transition between the structures of types I-fs and II-fs, we wrote each zone with a different pulse energy $\left(E_{1}=80 \mathrm{~nJ}, E_{2}=0.4 \mu \mathrm{J}, E_{3}=0.8 \mu \mathrm{J}, E_{4}=1.2 \mu \mathrm{J}\right.$, 
Table 2. Fabrication Details of the Sample

\begin{tabular}{cccc}
\hline $\begin{array}{c}\text { Energy } \\
(\mu \mathrm{J})\end{array}$ & $\begin{array}{c}\text { Square } \\
\text { identifier }\end{array}$ & $\begin{array}{c}\Delta n_{\mathrm{e}}{ }^{\mathrm{a}} \\
\left(\times 10^{-3}\right)\end{array}$ & $\begin{array}{c}\Delta n_{\mathrm{o}}{ }^{\mathrm{a}} \\
\left(\times 10^{-3}\right)\end{array}$ \\
\hline 0.08 & 1 & $+0.0 \pm 0.2$ & $+0.0 \pm 0.2$ \\
0.40 & 2 & $+1.6 \pm 0.2$ & $+1.2 \pm 0.2$ \\
0.80 & 3 & $+2.2 \pm 0.2$ & $+2.2 \pm 0.2$ \\
1.20 & 4 & $+1.9 \pm 0.2$ & $+2.3 \pm 0.2$ \\
1.60 & 5 & $-2.8 \pm 0.2$ & $+0.9 \pm 0.2$ \\
2.14 & 6 & $-4.9 \pm 0.2$ & $-0.2 \pm 0.2$ \\
\hline
\end{tabular}

${ }^{\mathrm{a}}$ Before annealing.

$E_{5}=1.6 \mu \mathrm{J}, E_{6}=2.14 \mu \mathrm{J}$ as illustrated in Table 2). Each square was written utilizing a dedicated computer program, which controlled the translation stage and an electronic shutter used to regulate the irradiation time. The sample was moved along the direction of the laser polarization for $100 \mu \mathrm{m}$ at a constant speed of $60 \mu \mathrm{m} / \mathrm{s}$ while the shutter was open, and then translated in the orthogonal direction for $1 \mu \mathrm{m}$ with the shutter closed; this process was iterated 100 times.

After fabrication, the sample was positioned between two crossed polarizers and viewed under an optical microscope, in transmission mode, to verify the presence of laser-induced birefringence in the laser-processed regions. This optical inspection revealed that only the squares written at the highest energy levels (\#5 and \#6) transmit light and hence are birefringent. The laserinduced extraordinary $\left(\Delta \phi_{\mathrm{e}}\right)$ and ordinary $\left(\Delta \phi_{\mathrm{o}}\right)$ phase retardation was measured with a phase-stepping interferometric technique. After measuring the thickness, $t_{\mathrm{p}}$, of each directly written structure, in the direction of the propagation of light, using calibrated microscope images, the modification of the refractive index in the irradiated regions was calculated from

$$
\Delta \phi=\frac{2 \pi}{\lambda} t_{\mathrm{b}} \Delta n,
$$

where $\lambda$ is, in this case, the wavelength of the probing $\mathrm{He}-\mathrm{Ne}$ laser utilized in the interferometric setup $(633 \mathrm{~nm})$. From the experimental data, presented in Fig. 7, showing the refractive index change versus increasing pulse energy, it may be noticed that no index change is observed in the first structure (\#1), then $\Delta n$ grows monotonically with the pulse energy until a maximum positive value of $+2.2 \times 10^{-3}$ (Table 2 ). From \#5 the structures are observed to be birefringent and the extraordinary index change becomes strongly negative, reaching a minimum value of $-4.9 \times 10^{-3}$ in \#6, which, as an absolute value, is the largest index modification induced in this silica sample. On the contrary, the ordinary index change, which is still positive in \#5 becomes negative in $\# 6$, reaching a minimum value of $-0.2 \times 10^{-3}$. Indeed, the relative measurement of the refractive index confirmed the optical observations, showing that the first four structures of the sample (\#1, \#2, \#3 and \#4) are optically isotropic (type I-fs); whereas in the last two structures (\#5 \#6) significant birefringence is measured. In addition, it should be noted that, an abrupt change in the sign of the laser-induced index modification arises at the same threshold as the birefringence.

In order to investigate the stability of the anisotropic structures of type II-fs in comparison with the non-birefringent ones of type I-fs, an annealing experiment was performed. The sample of six directly written regions was heated at a rate of $3^{\circ} \mathrm{C}$ per minute, kept at $200^{\circ} \mathrm{C}$ for one $\mathrm{h}$ and cooled to room temperature at $1^{\circ} \mathrm{C}$
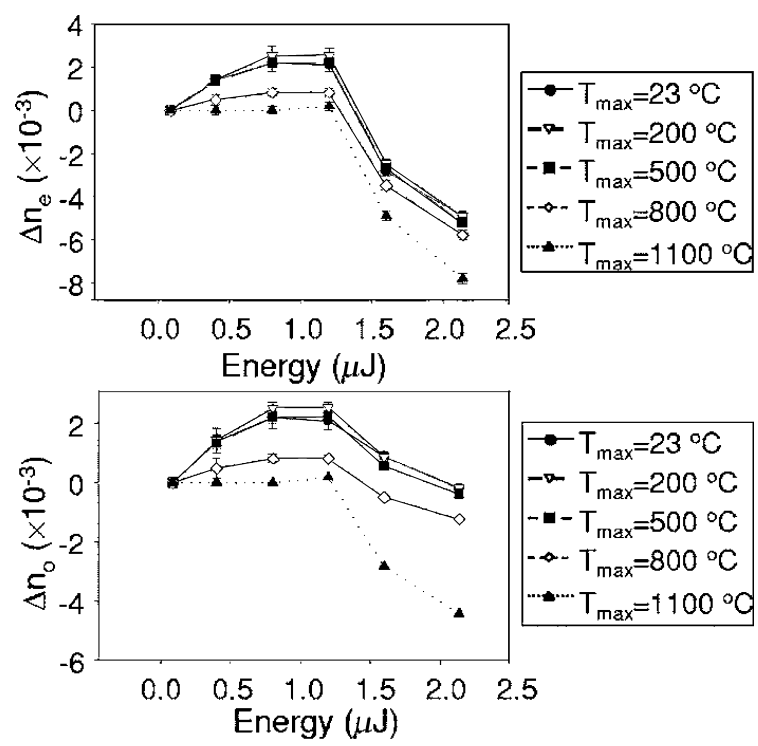

Fig. 7. Measurement of the laser induced index change of each directly written region vs the energy after each annealing step, (left) $\Delta n_{\mathrm{e}}$; (right) $\Delta n_{\mathrm{o}}$

per minute. The treatment was then repeated up to maximum temperatures of $500^{\circ} \mathrm{C}, 800^{\circ} \mathrm{C}, 1100^{\circ} \mathrm{C}$ and $1400^{\circ} \mathrm{C}$, and after each annealing step the sample was removed and the index change measured, except for the final case $\mathrm{T}=1400^{\circ} \mathrm{C}$, where the sample crystallized during the operation. Our results therefore refer to the steady state value assumed by the modified structures after being heated and then cooled back at room temperature (Fig. 7).

It has been observed that the index change of all the structures was unaltered up to a temperature of $500^{\circ} \mathrm{C}$, but above this temperature the isotropic structures belonging to type I-fs (\#1, \#2, \#3 and \#4) behaved very differently to the anisotropic structures of type II-fs (\#5 \#6). The index change of the isotropic nonbirefringent structures decreased by a factor of $\sim 0.4$, with respect to the initial value after heating to $800^{\circ} \mathrm{C}$, and finally disappeared after annealing at $1100^{\circ} \mathrm{C}$. On the contrary, the birefringent regions were still clearly visible under an optical microscope even after annealing at $1100^{\circ} \mathrm{C}$. Rather unexpectedly, however both the ordinary and extraordinary index change in the birefringent structures becomes more negative with respect to the unprocessed silica (Fig. 7). This behavior is more obvious in Fig. 8(a), where the normalized extraordinary index change, measured after each annealing step, is plotted versus the maximum temperature. It should be noted that, while for the structures of type I-fs the index change decreases after $500^{\circ} \mathrm{C}$, for the two structures of type II-fs, an increase is measured. These observations lead to the surprising conclusion that, in the anisotropic structures of type II-fs the absolute value of the modification of the refractive index induced by the laser, increases with the annealing temperature. Yet, the birefringence of the structures $\beta=\left|n_{\mathrm{e}}-n_{\mathrm{o}}\right|$ decreases with the temperature (Fig. 8(b)). Using the experimental measurement of the extraordinary and ordinary refractive index modification of the directly written regions $\left(\Delta n_{\mathrm{e}}\right.$ and $\Delta n_{\mathrm{o}}$ respectively), the values of the local index change in the nano-gratings $\Delta n_{1}=n_{1}-n_{\mathrm{bg}}$, and $\Delta n_{2}=n_{2}-n_{\mathrm{bg}}$ were calculated from $^{19)}$ 
(a)

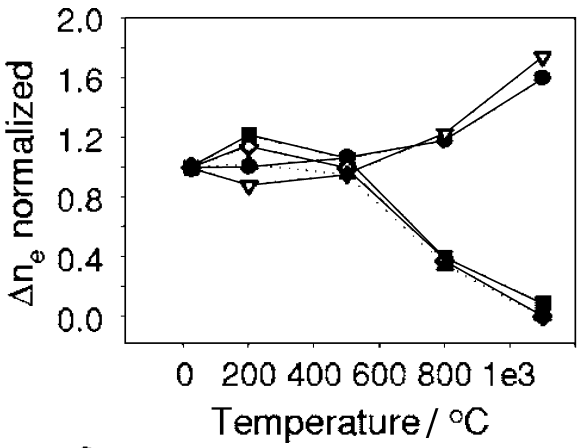

(b)

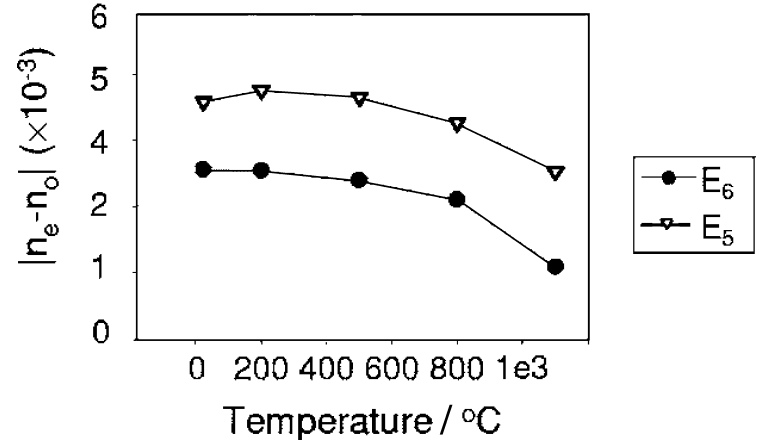

Fig. 8. (a) Extraordinary index change normalized to the value measured before annealing, vs the temperature. (b) Birefringence vs the temperature.

$$
\begin{aligned}
& \Delta n_{2}=\left[\sqrt{\frac{n_{1}{ }^{2} n_{2}{ }^{2} n_{2}^{2}+(1-f) n_{1}{ }^{2}}{n^{2}}}-n_{\mathrm{bg}}\right], \\
& \Delta n_{2}=\left[\sqrt{f n_{1}^{2}+(1-f) n_{2}^{2}}-n_{\mathrm{bg}}\right]
\end{aligned}
$$

where $n_{\text {bg }}$ is the refractive index of silica, $n_{1}$ and $n_{2}$ are the local refractive indices of the nano-grating, $f=t_{1} / \Lambda$ is the filling factor, $\Lambda=t_{1}+t_{2}$ is the period of the nano-grating, and finally, $t_{1}$ and $t_{2}$ are the width of the regions with index $n_{1}$ and $n_{2}$ respectively (Fig. 1). The value of the filling factor, $\mathrm{f}=0.3$, was chosen from our previous experimental measurements of the period of the nano-grating, created in various structures, which were written under similar conditions to the test sample here. Indeed, as it might be expected, both values of the local index change in the nano-gratings $\left(\Delta n_{1}\right.$, and $\left.\Delta n_{2}\right)$ decrease, in their absolute values, with increasing temperature (Fig. 9), hence the grating becomes weaker and, consequently, the value of the birefringence reduces, as shown in Fig. 8(b). However, since $n_{2}$ anneals more rapidly than $n_{1}$, the relative weight in (5) is such that the absolute values of the ordinary and extraordinary index change increase.

It was speculated that the different annealing rate within the nano-grating, which was deduced from the calculation of the local refractive indices versus increasing temperature, is related to the different local composition of the periodic regions of refractive index $n_{1}$ and $n_{2}$. The results reported in reference 6 , indeed revealed that the thin regions of width $t_{1}$, characterized by a strong negative index change with respect to silica (up to -0.1), are oxygen-deficient regions. However, such a structural modification is not detected in the regions of width $t_{2}$, which are characterized by a positive index change.

Although the mechanisms responsible for the creation of the femtosecond induced index change are still under investigation, it is believed that the positive index change, measured in the

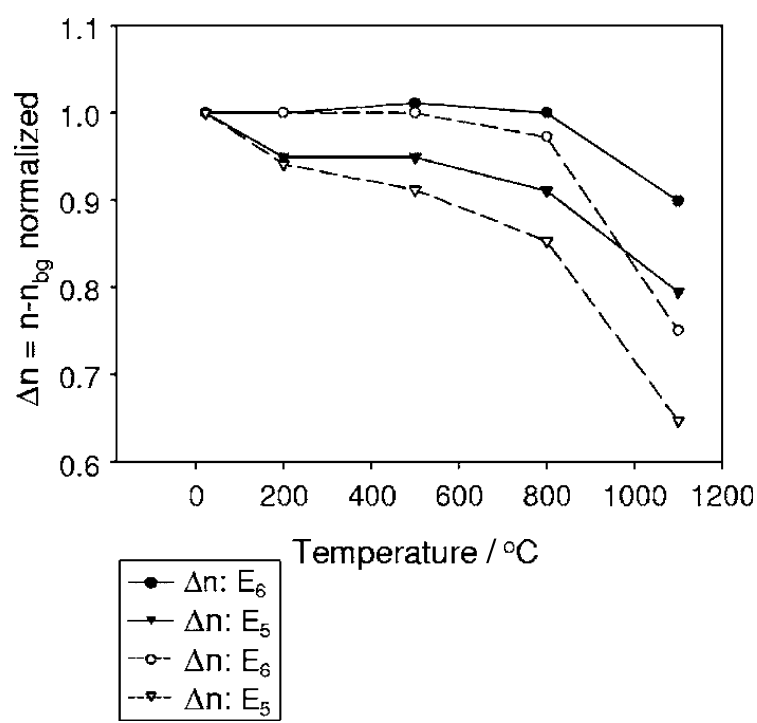

Fig. 9. $\Delta n_{1}$ and $\Delta n_{2}$ vs temperature rise and normalized to their initial value (before annealing).

structures of type I-fs, is associated to volume change, photoelasticity and to the modification of the absorption spectrum due to the presence of defects. ${ }^{22)}$

This type of modification eventually anneals at elevated temperatures. The regions of index $n_{2}$ have a similar thermal behaviour, whilst the strong structural modification of the regions of index $n_{1}$ prevents them from disappearing. ${ }^{16)}$

\section{Effect of writing laser parameters on nanostruc- tures formation}

Two different types of laser sources have been used in the experiments on the investigation of the effect of writing laser parameters on nanostructures formation. ${ }^{17)}$ The first laser source was a regeneratively amplified mode-locked Ti: Sapphire laser system (Coherent RegA) emitting a train of pulses with pulse duration varied from 150 to $250 \mathrm{fs}$, at repetition rate of $250 \mathrm{kHz}$ and at wavelength tunable from $750 \mathrm{~nm}$ to $850 \mathrm{~nm}$. Prior to focusing, the laser beam was linearly polarized along the $x$ - or y-axis (shown in Fig. 10 (a)) using a half-wave plate, and the pulse energy $\left(E_{\mathrm{p}}\right)$ was varied using a variable neutral density filter from $50 \mathrm{~nJ}$ to $1 \mu \mathrm{J}$. The laser beam was focused via a 50 $\mathrm{X}$ objective $(\mathrm{NA}=0.55) \sim 200 \mu \mathrm{m}$ below the surface of the 1-mm thick fused silica (Herasil 1). Straight lines were written by translating the sample along the $y$-axis, perpendicular to the propagation direction of the laser beam ( $z$-axis), as shown in Fig. 10(a). The second laser source was a variable repetition rate fiber laser system (IMRA America FCPA $\mu$ Jewel D-400). Using a similar experimental setup, the sample was irradiated under various repetition rates $(200 \mathrm{kHz}, 500 \mathrm{kHz}$ and $1 \mathrm{MHz})$ and at two different wavelengths (1045 $\mathrm{nm}$ and $522 \mathrm{~nm}$ ). The pulse duration was 400 fs and the beam was focused via a $30 \mathrm{X}$ single element aspheric lens (1045-nm processing) and a $40 \mathrm{X}$ microscope objective (522-nm processing), both at an operating NA of $\sim 0.32$.

As depicted in Fig. 10(a), only the line JM3 $x$ written with the polarization along $x$-axis (Fig. 10(b)), in the plane of observation $(x z)$ revealed the periodic modulation of the index change (for these structures, $x z$ is the plane perpendicular to the plates forming the nanogratings). On the contrary, the corresponding line JM3y, written with the same conditions, but with the polarization along $y$-axis (Fig. 10(b)) shows a homogeneous profile (for these 
structures, $x z$ is the plane parallel to the plates forming the nanogratings). Figure 10(c) is a close-up of part of Fig. 10(b) revealing that the filling factor $(f)$ is $\sim 0.3$, twice as high as the results reported in ref.6. In addition, the image demonstrates an abrupt change of density between the stripes (i.e. the zones of refractive index $n_{1}$ in Fig. 10(a)), and the surrounding regions (i.e. the zones of refractive index $n_{2}$ in Fig. 10(a)), suggesting that the mechanism leading to the modulation of the refractive index is highly nonlinear. As a further analysis, it was studied how the self-assembled periodic structures arrange themselves across adjacent written zones in the plane perpendicular to the propagation of the writing laser. For this experiment, a Fresnel zone plate B (FZPB), described in ref. 8 was chosen, as it offered a wide area to be imaged under the SEM and was written with similar conditions to sample JM3. The silica plate containing the FZP was polished in the plane perpendicular to lens' axis ( $x y$

(a)

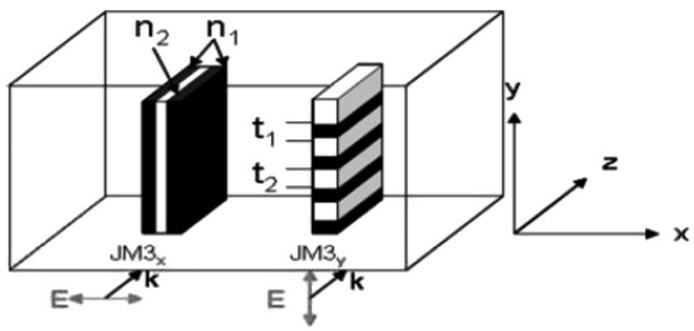

(b)

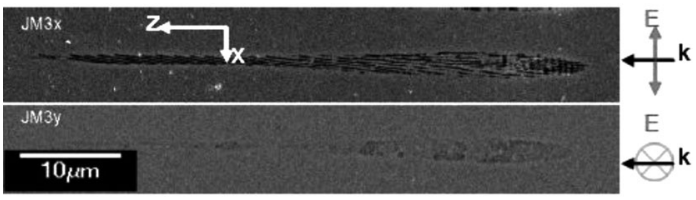

(c)

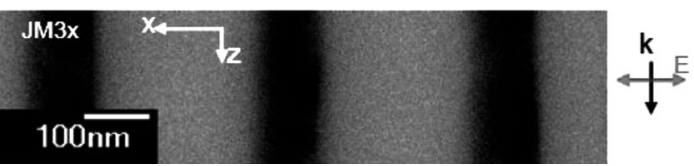

Fig. 10. Modified regions in sample JM3. E: electric field of the writing laser, $k$ : wave vector of the writing laser beam, $\mathrm{JM} 3_{x}$ and $\mathrm{JM} 3_{y}: E_{\mathrm{p}}=$ $0.48 \mu \mathrm{J}, V=200 \mu \mathrm{m} / \mathrm{s}, \lambda=850 \mathrm{~nm}$. (a): Schematic of the sample JM3 showing how the self-assembled periodic structures are expected to be arranged. $n_{1}$ and $n_{2}$ : local refractive indices of the plates of thickness $t_{1}$ and $t_{2}$, respectively. (b): SEM images of $\mathrm{JM} 3_{x}$ and $\mathrm{JM} 3_{y}$ in the $x z$, plane. (c): Detail of the subwavelength periodic structure formed in $\mathrm{JM} 3_{x}$.

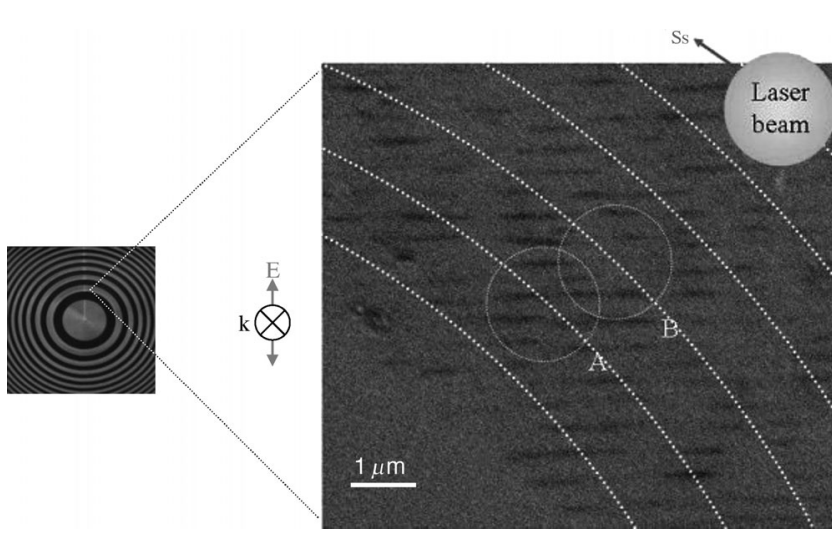

Fig. 11. SEM image of the nanogratings in the Frenel zone plate B [8]. $E$ : electric field of the writing laser, $k$ : wave vector of the writing laser beam, Ss: movement direction of the translation stage. plane) until the direct-written Fresnel rings were brought to the surface. Then, after being coated with a thin gold layer, the sample was analyzed with an SEM (JSM-5910) in backscattering emission mode. Figure 11 shows how the laser processed zones are easily distinguished from pure silica by the presence of the nanostructures. As expected, the grating ruling is perpendicular to the direction of the polarization of the writing laser beam. In addition, the image clearly shows that the selfassembled nanostructures formed in adjacent lines are aligned. As described in ref.8, the Fresnel rings were realized by writing circles with a space between them smaller than the writing resolution; hence the processed zones are formed by a series of partially overlapped directly written circles (Fig. 11). Given the geometry of the lens and the speed of the translation stage, the points in B in Fig. 11 were written $\sim 2$ s after the points in A. Despite the long time elapsing between the formation of the periodic structures in the two regions, the nanograting is continuous across the two written lines. This evidence suggests that the structure imprinted in a line provides an initial "seeding" condition during the formation of the self-organized periodic assembly in the adjacent line. This is a very important property as it demonstrates the ability of realizing periodic nanostructures over a larger area, rather than being limited to the size of the focused spot.

Figure 12 presents SEM images of the modified region in the $x z$ plane induced by three different writing laser wavelengths. The nanogratings, clearly visible in Fig. 12, are only in the head of the modified regions. No periodic structures can be observed in the tails of the features as previous shown in Fig. 10(b). The reasons leading to this noticeable difference is still under investigation, which might be related to the different etching solutions. Besides the main periodicity $\Lambda_{\mathrm{E}}$ which is in the direction of the polarization of the laser beam (E), a second periodicity $\Lambda_{\mathrm{k}}$ is identified perpendicular to $\Lambda_{\mathrm{E}}$, and hence parallel the direction of propagation of laser beam (k), as shown in Fig. 12. Furthermore, $\Lambda_{\mathrm{k}}$ is $660 \pm 50 \mathrm{~nm}$ at $1045 \mathrm{~nm}$ (Fig. 12(a)), $551 \pm 20 \mathrm{~nm}$ at $800 \mathrm{~nm}$ (Fig. 12(b)) and $326 \pm 50 \mathrm{~nm}$ at $522 \mathrm{~nm}$ (Fig. 12(c)) in the cropped area in the head of the modified region, which is very close to the ratio $\lambda / n$ in fused silica. This kind of periodic selfassembly behaves as a uniaxial form-birefringence material. This is also confirmed using the FCPA $\mu$ Jewel D-400 at wavelengths of $1045 \mathrm{~nm}$ and $522 \mathrm{~nm}$. Figure 13 shows a replica of IMRA's

(a)

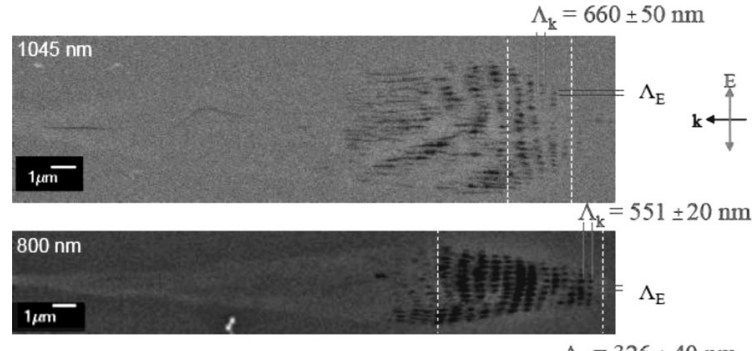

(c)

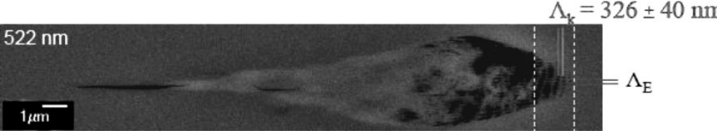

Fig. 12. SEM images of nanogratings formed by three different central wavelengths of the irradiated laser pulses in the $x z$ plane, $E$ : electric field of the writing laser, $k$ : wave vector of the writing laser beam. (a): $\tau \mathrm{p}=$ $400 \mathrm{fs}, E_{\mathrm{p}}=2 \mu \mathrm{J}, V=160 \mu \mathrm{m} / \mathrm{s}, R_{\mathrm{ep}}=200 \mathrm{kHz}$. (b): $\tau_{\mathrm{p}}=150 \mathrm{fs}, E_{\mathrm{p}}=0.5$ $\mu \mathrm{J}, V=100 \mu \mathrm{m} / \mathrm{s}, R_{\mathrm{ep}}=250 \mathrm{kHz}$. (c): $\tau_{\mathrm{p}}=400 \mathrm{fs}, E_{\mathrm{p}}=0.5 \mu \mathrm{J}, V=16$ $\mu \mathrm{m} / \mathrm{s}, R_{\mathrm{ep}}=200 \mathrm{kHz}$. 

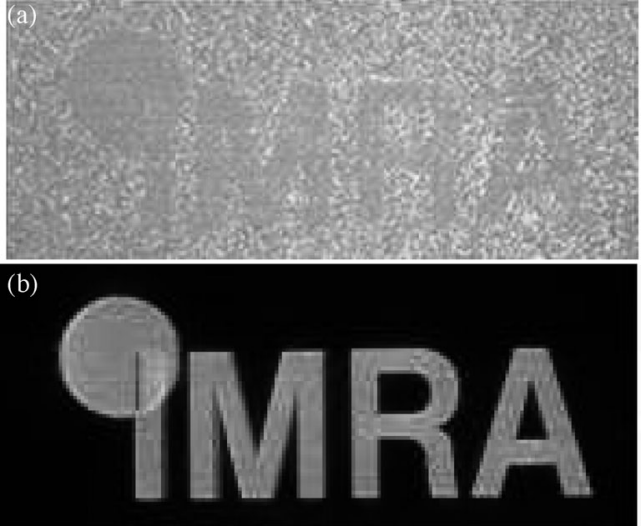

Fig. 13. Raster scanned replica of IMRA icon, $10-\mu \mathrm{m}$ line spacing, $500-\mathrm{kHz}$ repetition rate, $1045-\mathrm{nm}$ wavelength, $1.5-\mu \mathrm{J}$ pulse energy, $\sim 12$-h process time, $\sim 10$-mm icon width. (a) without cross-polarizers, (b) with cross-polarizers, (c) SEM of line cross-section.

(a)

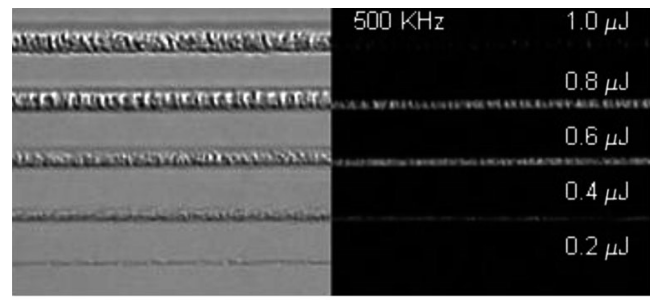

(b)

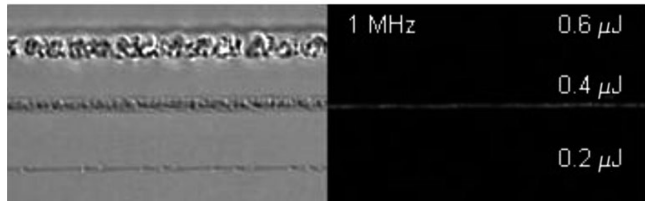

Fig. 14. Microscope images of the irradiated regions in the $x y$ plane, image on the left taken by back-illumination without polarizers, image on the right taken by back-illumination with cross polarizers of the same irradiated area. (a): $R_{\mathrm{ep}}=500 \mathrm{kHz}, V=500 \mu \mathrm{m} / \mathrm{s}$. (b): $R_{\mathrm{ep}}=1 \mathrm{MHz}, V=$ $1000 \mu \mathrm{m} / \mathrm{s}$. (a)
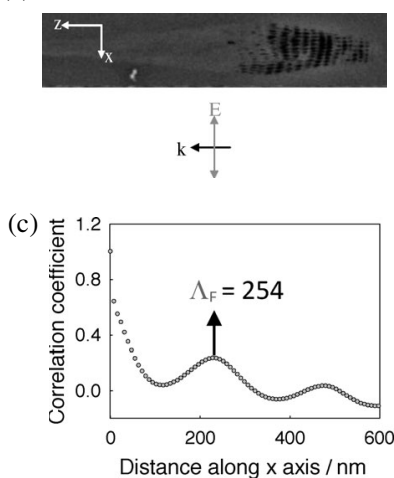

(b)
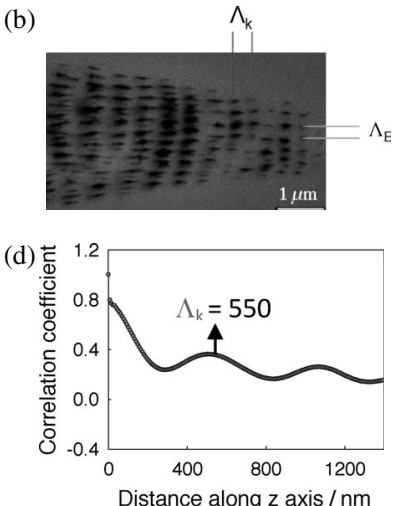

Fig. 15. (a) SEM image of a written line in the $x z$ plane, $E$ : electric field of the writing laser, $k$ : wave vector of the writing laser beam $(\lambda=$ $800 \mathrm{~nm}, \tau_{\mathrm{p}}=150 \mathrm{fs}, E_{\mathrm{p}}=0.5 \mu \mathrm{J}, V=100 \mu \mathrm{m} / \mathrm{s}, R_{\mathrm{ep}}=250 \mathrm{kHz}$ ). (b) Cropped head of the modified region used for calculation. Corresponding correlation functions calculated along the $x$ (b) and $z$ (c) axes. icon that was made by raster-scanning closely spaced lines (10 $\mu \mathrm{m}$ spacing). ${ }^{17)}$ Generally, increased repetition rate allowed for processing at higher scan speeds. However, when the repetition rate of the laser pulses reaches $500 \mathrm{kHz}$ and Ep is over $\sim 1 \mu \mathrm{J}$ (Fig. 14(a)), or when the repetition rate reaches $1 \mathrm{MHz}$ and Ep

(a)

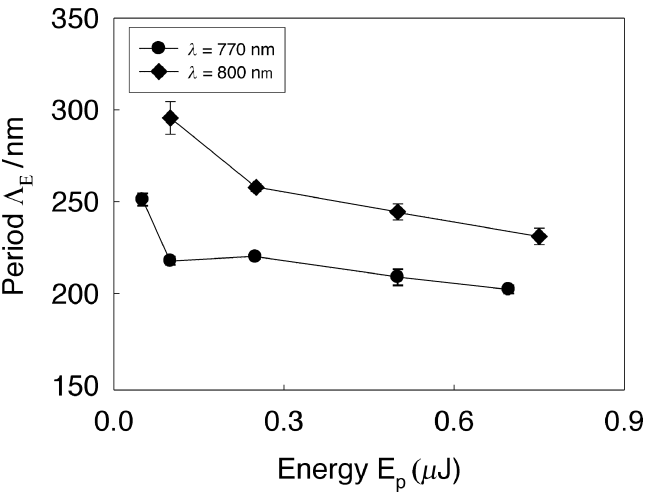

(b)

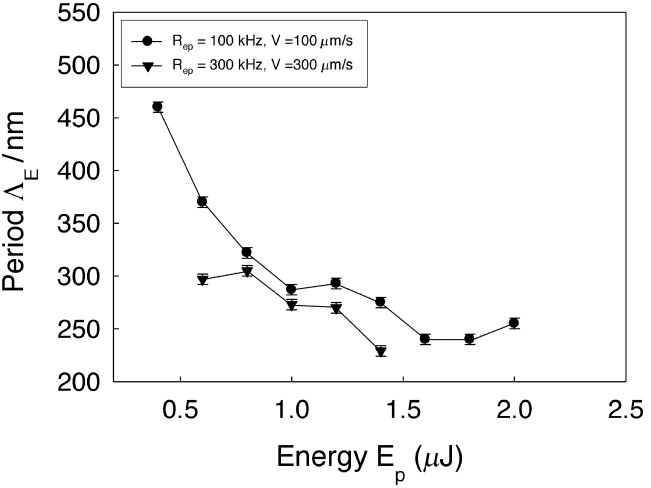

(c)

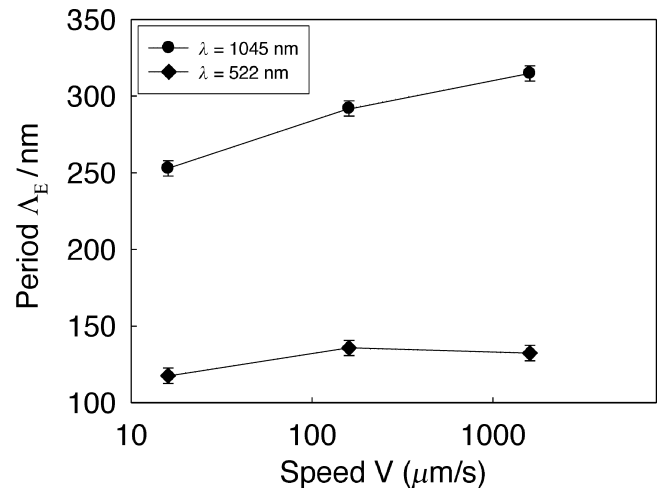

(d)

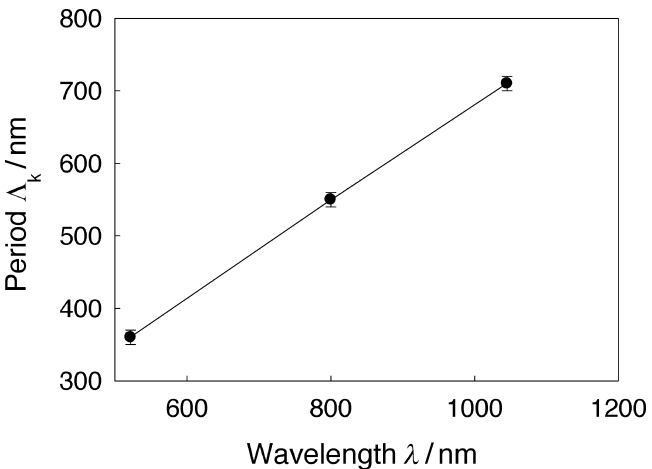

Fig. 16. (a) Periodicity $\Lambda_{\mathrm{E}}$ versus $E_{\mathrm{p}}\left(\tau_{\mathrm{p}}=150 \mathrm{fs}, R_{\mathrm{ep}}=250 \mathrm{kHz}, V=\right.$ $100 \mu \mathrm{m} / \mathrm{s})$. (b) Periodicity $\Lambda_{\mathrm{E}}$ versus $E_{\mathrm{p}}(\lambda=1045 \mathrm{~nm})$. (c) Periodicity $\Lambda_{\mathrm{E}}$ versus speed $V$ at two different wavelengths. (d) Periodicity $\_k$ versus three different wavelengths. 
is over $\sim 0.6 \mu \mathrm{J}$ (Fig. 14(b)), no birefringence or nanogratings could be observed in the irradiated regions of the sample. This could be explained by the occurrence of an accumulation effect within the focal volume when the pulse repetition rate is greater than $\sim 500 \mathrm{kHz} .^{18), 19)}$ This indicates that the pulse energy range to produce self-assembled nanogratings narrows when the pulse repetition rate of the laser increases.

However, when the repetition rate of the laser pulses reaches $500 \mathrm{kHz}$ and Ep is over $\sim 1 \mu \mathrm{J}$ (Fig. 14(a)), or when the repetition rate reaches $1 \mathrm{MHz}$ and $E_{\mathrm{p}}$ is over $\sim 0.6 \mu \mathrm{J}$ (Fig. 14(b)), no birefringence or nanogratings could be observed in the irradiated regions of the sample. This could be explained by the occurrence of an accumulation effect within the focal volume when the pulse repetition rate is greater than $\sim 500 \mathrm{kHz} .^{23), 24)}$ This indicates that the pulse energy range to produce self-assembled nanogratings narrows when the pulse repetition rate of the laser increases. In order to quantitatively analyze the periodicity $\Lambda_{\mathrm{E}}$ and $\Lambda_{\mathrm{k}}$, an algorithm based on the correlation coefficients between different points in the SEM image versus their distance was developed. By calculating the average value of the periods from the first maximum of the correlation functions, periods along the $x$ and $z$ direction are obtained, shown in Fig. 15. Our analysis of numerous SEM images reveals that the periodicity $\Lambda_{\mathrm{E}}$ and $\Lambda_{\mathrm{k}}$ does not change with the scanning speed from $50 \mu \mathrm{m} / \mathrm{s}$ to $500 \mu \mathrm{m} / \mathrm{s}$. However, for $0<E_{\mathrm{p}}<1 \mu \mathrm{J}, \Lambda_{\mathrm{E}}$ decreases with increasing $E_{\mathrm{p}}$ (Fig. 16(a)); for $E_{\mathrm{p}}>1 \mu \mathrm{J}, \Lambda_{\mathrm{E}}$ increases with increasing $E_{\mathrm{p}}$ (Fig. 16(b)). In addition, the wavelength dependence illustrated in Fig. 16(a) suggests that shorter wavelengths yield significantly smaller structures; this is also confirmed in Fig. 16(c). Furthermore, $\Lambda_{\mathrm{k}}$ in the head of the laser-modified region does not change with the pulse energy or speed within our study range and is confirmed to be approximately equal to the laser wavelength (shown in Fig. 16(d)).

\section{Calligraphic femtosecond laser writing and self- assembled nanostructures}

During the study of the self-assembled nanostructures formation a remarkable phenomenon was observed manifested as a change in material modification, in particular in the nanostructures and related form birefringence, which can be controlled by reversing the writing direction. ${ }^{18)}$ The effect resembles writing with a quill pen and is interpreted in terms of the light pressure on electron plasma produced by a tilted front of the ultrashort laser pulse which results in an anisotropic heat current.

The laser radiation, in a Gaussian mode, produced by a regeneratively amplified mode-locked Ti: Sapphire laser (150-fs pulse duration, $250 \mathrm{kHz}$ ), operating at $800 \mathrm{~nm}$, was focused via a 50 $\mathrm{X}(\mathrm{NA}=0.55)$ objective into the sample. A series of lines were directly written by scanning in alternating directions at a depth of $0.5 \mu \mathrm{m}$ below the front surface. The writing speed was 200 $\mu \mathrm{m} / \mathrm{s}$ and each line was written with only one pass, in one direction, of the laser, with the polarization directed perpendicular to the line and pulse energy of $0.9 \mu \mathrm{J}$. After writing, the structures were side-polished and imaged with a SEM. The SEM images expose tracks elongated in the direction of light propagation due to beam's confocal parameter, and enhanced by self-focusing effects, with a periodic structure in the direction of light polarization (Fig. 17). On closer inspection it was observed a difference in the structures written in opposite directions. This difference is revealed in small variations of the length of the tracks and of a tilt of the periodic structures written in the forward and reverse directions.

In another experiment a series of lines was written using an

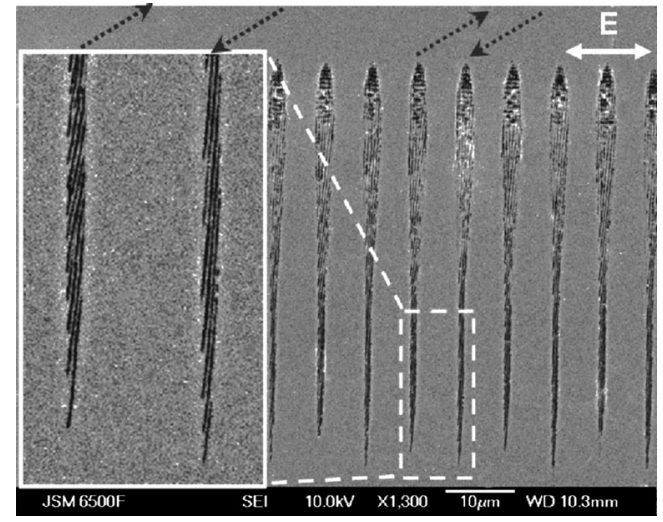

Fig. 17. SEM images of cross sections of the structures in glass along light propagation. The distance between lines is $7 \mu \mathrm{m}$.

IMRA-FCPA $\mu$ Jewel D-400 amplified ytterbium fiber laser system, operating at $1045 \mathrm{~nm}$, with pulse duration $<500 \mathrm{fs}$ and repetition rates ranging from $100 \mathrm{kHz}$ to $1 \mathrm{MHz}$. The high stability of the FCPA laser system is crucial for systematic studies. The polarization of the laser was aligned perpendicular to the writing direction. The lines were written in alternating directions from forward to reverse and using different pulse energies ranging from 0.2 to $1.8 \mu \mathrm{J}$. After writing, microscope images were captured using both crossed-polarized (CP) and Nomarski-DIC illumination (both back-illumination). Composite images were created which show the same portion of each feature using the two illumination techniques. With these composite images, the amount of birefringence visible with the $\mathrm{CP}$ illumination can be compared with the texture of the feature using the DIC imaging technique. In one of the experiments 10 groups of 4 lines was written with alternating writing direction and each series with different repetition rate and speed. The product of repetition rate and writing speed was kept constant, in order to maintain the same total fluence. Lines written in both directions at low energies were the same (Fig. 18). However, with an increase in energy it was observed the appearance of directional dependence in the written. lines, which was strongest at about $0.8-0.9 \mu \mathrm{J}$. The directional dependence is more clearly seen in the birefringence of the lines This dependence can also be observed in the morphology (texture) of lines written in opposite directions, with a line written in one direction being rougher than a line written in the reversed direction (Fig. 18a). However, with further increase of energy above a certain threshold value, both lines become uneven with indications of collateral damage, and the birefringence of the lines disappears as well as the directional dependence. The latter phenomenon can be explained by a cumulative thermal effect. This is supported by the presence of modifications with rough features, much bigger than the spot of the beam, at high repetition rates $(500 \mathrm{kHz}-1 \mathrm{MHz})$ and the absence of such features with collateral damage at low repetition rates (below $300 \mathrm{kHz}$ ). This agrees with the heat diffusion time of about $1-\mu \mathrm{s}$. The dependence of the effect was also tested on writing speed near the energy threshold of the disappearance of directional phenomenon and observed that the directional dependence strengthen at lower writing speeds (Fig. 18b).

When the beam was turned by $90^{\circ}$, using a two-mirror periscope, and other writing parameters maintained the same including the polarization of the laser beam perpendicular to the stage movement, it was observed only weak difference in structures 
(a)

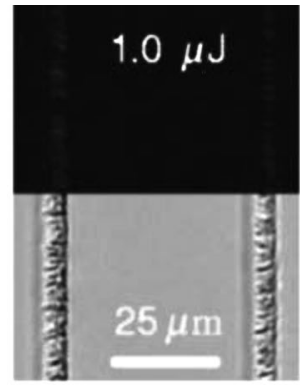

(b)

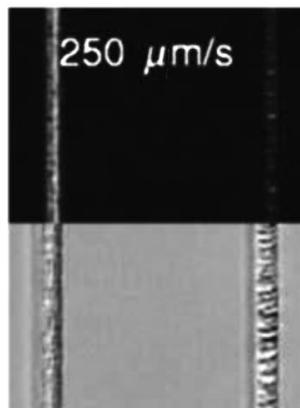

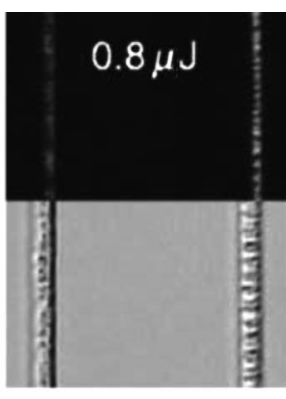

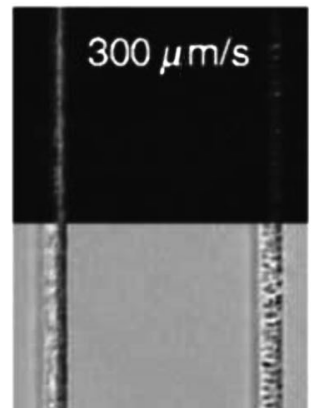

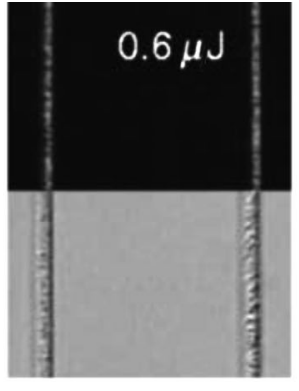

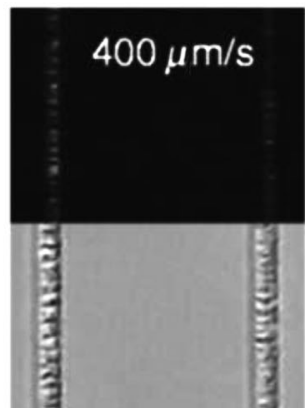

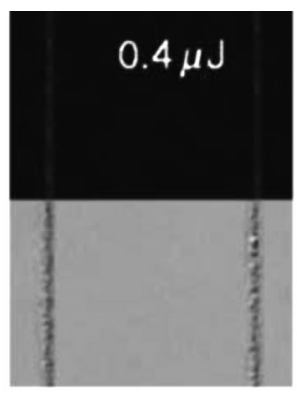

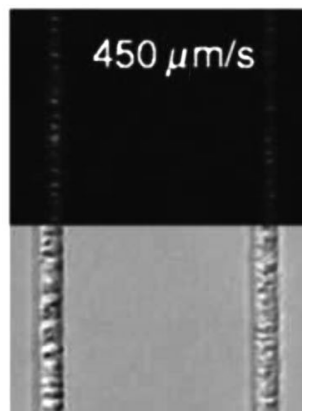

Fig. 18. Images in crossed polarizers (dark part) and Nomarski-DIC (light part) of the lines written in glass in opposite directions at repetition rate of $500 \mathrm{kHz}$ with (a) writing speed of $500 \mu \mathrm{m} / \mathrm{s}$ and different energies and (b) pulse energy of $0.9 \mu \mathrm{J}$ and different writing speeds.

written in opposite directions. This indicates that an asymmetry in the structure of the beam can be responsible for the observed phenomenon.

An intriguing result is the observation of different textures in the processed material for laser polarizations perpendicular and parallel to the movement of the sample in one direction and the same textures for two polarizations when writing in the opposite direction (Fig. 19a). The SEM images of the cross-sections of the lines, along the light propagation, revealed a different texture in the lines written in opposite directions (Fig. 19b). Remarkably, the nanograting of about $300 \mathrm{~nm}$ period, which is responsible for the form birefringence of irradiated regions, can be seen only in the initial part of cross sections of lines written in one of two directions. This small area is followed by one with a collateral damage due to thermal effect, which correlates with a weak birefringence of these lines. It was also observed, that in almost entire cross sections of the lines, written in opposite direction, there is the nanograting along the direction of light polarization with the period of about $250 \mathrm{~nm}$ together with the additional periodicity, along the direction of light propagation, of about $720 \mathrm{~nm}$, which is of the wavelength of light ( $\lambda / n, \lambda=1045 \mathrm{~nm}, n=1.45)$ (Fig. $19 \mathrm{~b})$. These lines demonstrate no evidence of the collateral thermal damage and much stronger birefringence (Fig. 19a).

The writing anisotropy is observed only at particular pulse energies, which excludes the stage movement as the cause. Inspection of the intensity distribution of the laser beam did not reveal any peculiarities in the shape of the beam, which was close to circular (Gaussian shape). The only possibility left to explain the puzzle of the writing direction anisotropy is related to the anisotropy of the frequency distribution (frequency chirp) in the beam. A spatial frequency chirp and related pulse front tilt is quite common in femtosecond laser systems. ${ }^{24)}$ Even a small delay across the beam that corresponds to $\sim 10 \%$ of the pulse duration results in pulse tilt as strong as tens of degrees in the vicinity of the focal plane. The pulse front tilt is enhanced in a dispersive media, as in the case of electron plasma close to (a)

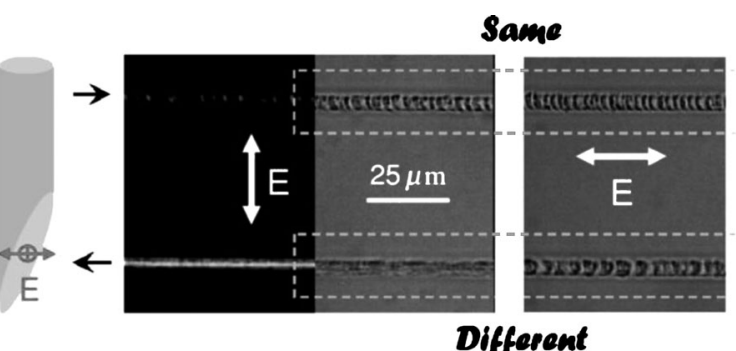

(b)

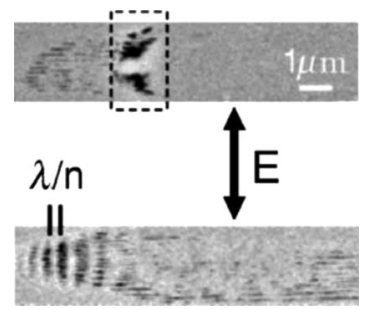

Fig. 19. (a) CP and DIC images of the lines written with orthogonal polarizations with $500 \mathrm{kHz}$ repetition rate, writing speed of $250 \mu \mathrm{m} / \mathrm{s}$, and pulse energy of $0.9 \mu \mathrm{J}$. The difference in texture for two polarizations is observed only for one writing direction. The tilted front of the pulse along writing direction is shown. (b) SEM images of cross sections of lines written with polarization perpendicular to writing direction are also shown. The region of collateral damage is marked with a black dashed line.

plasma frequency, which is formed in the focus of the beam due to multiphoton ionization of glass. The pulse front tilt is a tilt in the intensity distribution in the front of the pulse. It is known, that in the presence of intensity gradients, the charges (e.g. electrons) experience the pondermotive force (light pressure), which expels the electrons from the region of high intensity. ${ }^{26)}$ Indeed, free electrons are affected by a variation of the laser intensity as they quiver in the electric field of the laser pulse. The directional asymmetry of ultrashort-pulse-light-induced modifications in glass does not depend on the orientation of a medium or the 
direction of light propagation and can be controlled by the tilt of a pulse front, which is in contrast to analogous phenomenon in crystals. ${ }^{27)}$ It has been suggested that the ponderomotive force (light pressure) at the front of the pulse is responsible for the phenomenon. Due to the tilt of the intensity distribution, the force will act on the electron plasma along the direction of the intensity gradient. A heat current $J$, which is carried by the electrons of plasma created by the femtosecond laser pulse, and generated by the ponderomotive force and the photon drag effect, can be phenomenologically presented in the following form:

$$
J_{\mathrm{i}}=\eta_{\mathrm{ijklmn}} E_{\mathrm{j}} E_{\mathrm{k}}^{*} \nabla_{\mathrm{n}}\left(E_{1} E_{\mathrm{m}}^{*}\right)+i \zeta_{\mathrm{j} \mathrm{jk} l m n} E_{\mathrm{j}} E_{\mathrm{k}} E_{1}^{*} E_{\mathrm{m}}^{*} k_{\mathrm{n}}
$$

where subscripts label Cartesian indices, $E$ is the complex amplitude of the light electric field, i. e. $E_{\mathrm{k}} E_{1}^{*}$ is proportional to the light intensity and is responsible for heating via the plasma absorption. The first and second term in the right-hand-side of Eq. (6) describe pressure created by the front of the pulse and photon drag effect, respectively, $\eta_{\mathrm{ijk} \mid m n}$ and $\zeta_{\mathrm{ijk} l m n}=\zeta_{\mathrm{ikjlmn}}=\zeta_{\mathrm{ilmjkn}}$ are sixth rank tensors, $k$ is wave vector. Movement of the beam along the heat current will produce stronger heating of the medium which results in melting and disappearance of the nanograting and the related form birefringence in the writing direction corresponding to the pulse front tilt, which was referred as the "quill effect." 18)

\section{Conclusions}

We point out in this review that the femtosecond laser induced nanograting is also associated with a negative effective index change, and we provide a consistent explanation for the negative birefringence associated with type II-fs structures, using a model of form birefringence.

Experiment related to the annealing of femtosecond directly written structures, showed extraordinary thermal stability of type II-fs structures. The unexpected behavior displayed by these structures, namely a growth of the absolute value of the ordinary and extraordinary refractive index change with the temperature increase, was justified considering the periodical structure formed by the femtosecond laser in the irradiated volume. Nonbirefringent regions started degrading at $800^{\circ} \mathrm{C}$; whereas the femtosecond written structures of type II-fs could not be annealed, making them an attractive candidate for embedded polarization sensitive photonic applications, and in particular for volumetric optical memory.

Two different types of periodic structures have been identified from crosssectional SEM images of femtosecond pulse-modified regions in fused silica. The pulse energy range that produces nanogratings narrows when the pulse repetition rate of the writing laser increases. The periodicity $\Lambda_{\mathrm{E}}$ and $\Lambda_{\mathrm{k}}$ increase with increasing wavelength of the writing laser and $\Lambda_{\mathrm{k}}$ in the head of the modified region remains $\sim \lambda / \mathrm{n}$ in fused silica. The spatial coherence of these nanogratings in the plane perpendicular to the beam propagation direction has also been demonstrated.

It is remarkable that the research on the self-assembled nanostructures and form birefringence produced by femtosecond laser writing has led to the discovery of another remarkable phenomenon which is femtosecond laser calligraphic writing. Moreover, modifications of materials by light span from photosynthesis and photography to material processing and laser writing and there are only few parameters of the light beam which control material transformations, in particular wavelength, intensity, exposure time and pulse duration. Our results add one more parameter to this list - direction of beam movement or pulse front tilt. It was anticipated that this type of light-matter interaction, sensitive to the direction of movement of a laser beam will open new opportunities in direct writing, material processing, optical trapping, and manipulation.

Acknowledgement The support of the Engineering and Physical Sciences Research Council (EPSRC) and New Energy and Industrial Technology Development Organization (NEDO) is acknowledged.

\section{References}

1) K. Miura, J. Qiu, H. Inouye, T. Mitsuyu and K. Hirao, Appl. Phys. Lett., 71, 3329-3331 (1997).

2) C. B. Schaffer, A. Brodeur and E. Mazur, Meas. Sci. Technol., 12, 1784-1794 (2001).

3) G. Cerullo, R. Osellame, S. Taccheo, M. Marangoni, D. Polli, R. Ramponi, P. Laporta and S. D. Silverstri, Opt. Lett., 27, 1938-1940 (2002).

4) E. Glezer, M. Milosavljevic, L. Huang, R. J. Finlay, T. H. Her, J. P. Callan and E. Mazur, Opt. Lett., 21, 2023-2025 (1996).

5) H. Sun, Y. Xu, S. Juodkazis, K. Sun, M. Watanabe, S. Matsuo, H. Misawa and J. Nishii, Opt. Lett., 26, 325-327 (2001).

6) P. G. Kazansky, H. Inouye, T. Mitsuyu, K. Miura, J. Qiu, K. Hirao and F. Starrost, Phys. Rev. Lett., 82, 2199-2202 (1999).

7) L. Sudrie, M. Franco, B. Prade and A. Mysyrowicz, Opt. Commun., 191, 333-339 (2001).

8) E. Bricchi, J. D. Mills, P. G. Kazansky, B. G. Klappauf and J. J. Baumberg, Opt. Lett., 27, 2200-2202 (2002).

9) J. D. Mills, P. G. Kazansky, E. Bricchi and J. J. Baumberg, Appl. Phys. Lett., 81, 196-198 (2002).

10) Y. Shimotsuma, P. G. Kazansky, J. Qiu and K. Hirao, Phys. Rev. Lett., 24, 2474051 (2003).

11) Y. Shimotsuma, K. Hirao, J. Qiu and P. G. Kazansky, Modern Physics Letters B, 19, 225-238 (2005).

12) B. C. Stuart, M. D. Feit, A. M. Rubenchik, B. W. Shore and M. D. Perry, Phys. Rev. Lett., 74, 2248-2251 (1995).

13) E. Bricchi, B. G. Klappauf and P. G. Kazansky, Opt. Lett., 29, 119-121 (2004).

14) C. Hnatovsky, R. S. Taylor, P. P. Rajeev, E. Simova, V. R. Bhardwaj, D. M. Rayner and P. B. Corkum, Appl. Phys. Lett., 87, 014104 (2005).

15) E. Bricchi and P. G. Kazansky, Appl. Phys. Lett., 88, 111119 (2006).

16) C. Hnatovsky, R. S. Taylor, E. Simova, V. R. Bhardwaj, D. M. Rayner and P. B. Corkum, Opt. Lett., 30, 1867-1869 (2005).

17) J. Yang, E. Bricchi, P. G. Kazansky, J. Bovatsek and A. Y. Arai, Opt. Express, 14, 10117-10124 (2006).

18) P. G. Kazansky, W. Yang, E. Bricchi, J. Bovatsek, A. Arai, Y. Shimotsuma, K. Miura and K. Hirao, Appl. Phys. Lett., 90, 151120 (2007).

19) M. Born and E. Wolf, "Principles of Optics", 7th ed. by Cambridge U. Press, Cambridge (1999) pp. 827-840.

20) S. J. Mihailov, C. W. Smelser, D. Grobnic, R. B. Walker, P. $\mathrm{Lu}, \mathrm{H}$. Ding and J. Unruh, J. Lightwave Technol., 22, 94-100 (2004).

21) P. G. Kryukov, Yu V. Larionov, A. A. Rybaltovskii, K. A. Zagorulko, A. Dragomir, D. N. Nikogosyan and A. A. Ruth, Microelectron. Eng., 69, 249-255 (2003).

22) J. W. Chan, T. Huser, S. Risbud and D. M. Krol, Opt. Lett., 26, 1726-1728 (2001)

23) C. B. Schaffer, J. F. Garcia and E. Mazur, Applied Physics AMaterials Science \& Processing, 76, 351-354 (2003).

24) C. M. Eaton, H. B. Zhang and P. R. Herman, Opt. Express, 13, 4708-4716 (2005).

25) S. Akturk, M. Kimmel, P. O'She and R. Trebino, Opt. Express, 11, 491-501 (2003).

26) W. L. Kruer, "The Physics of Laser Plasma Interactions," Addison-Wesley, Redwood City (1988) Vol. 73, p. 60.

27) W. Yang, P. G. Kazansky and Y. P. Svirko, Nature Photonics, 2, 99-104 (2008). 


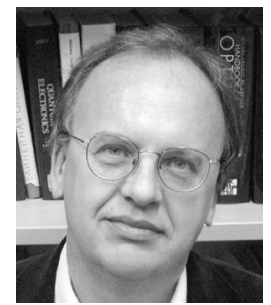

Peter G. Kazansky received a M. Sc. degree in Physics from Moscow State University in 1979 and a Ph. D. under supervision of Nobel Laureate for the invention of laser A.M. Prokhorov from the General Physics Institute in 1985. He was awarded the Leninskii Komsomol Prize in 1989 for the pioneering work on "Circular photogalvanic effect in crystals" (which concerns conversion of photon angular momentum to charge carrier momentum). From 1989 to 1993 he led a group in the GPI which unravelled the mystery of a new optical phenomenon - light-induced frequency doubling in centosymmetric media. In 1992 he joined the Optoelectronics Research Centre at the University of Southampton where since 2001 he is a chair leading "Physical Optics" group and pursuing his interests in new optical materials, photosensitivity, ultrafast material processing, nanotechnology and quantum information research. He is Vice-Chair of the TC-20 Technical Committee on Glasses for Optoelectronics of ICG, the International Commission on Glass.

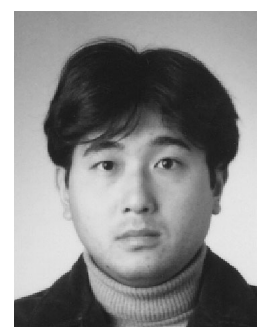

Yasuhiko Shimotsuma received B. Sc. and M. Sc. degrees in Engineering from Tohoku University in 1996. In 1996, he joined the Corporate R\&D Division for Components \& Devices of Kyocera Corporation. From 2004 to 2006, he has been a researcher in the Fukui Institute for Fundamental Chemistry of Kyoto University. In 2007 he joined NEDO Integrated Photonics Laboratory where he is an associate professor. His current area of interest is the physics of light-matter interaction and fabrication of the new optical devices by using the femtosecond laser. He is a member of CerSJ, JSAP, LSJ, OSA, and SPIE.

E-mail: yshimo@collon1.kuic.kyoto-u.ac.jp 